\title{
Grafting in rhinoplasty: correlation between the size of nasal bone and the amount of septal cartilage available for harvesting
}

\begin{abstract}
Importance: Predicting the amount of septal cartilage available for harvesting is still a challenge for Rhinoplasty Surgeons. A preoperative clinical evaluation would be of great value if capable of predicting that amount for surgical planning.

Objective: To correlate the measures between the size of the nasal bone and the amount of septal cartilage available for harvesting intraoperatively.

Design: We performed nasal size measures in 30 participants (aged 16-45years). The nasal bone was palpated and its size was measured with a ruler (measure 1). Dorsal nasal length (measure 2), was also obtained through palpation and measured with a ruler. The amount of septal cartilage available for harvesting was estimated by 2 intraoperative measures: distance in straight line from the rhinion to the anterior septal angle (measure 3 ), and distance in straight line from the osteo-cartilaginous transition to the caudal septal midpoint (measure 4). Our theory is that patients with shorter nasal bones would have greater amount of septal cartilage available for harvesting, based on the premise that if the nasal bone is short, greater amounts of cartilage will make up for the rest of nasal septum length.
\end{abstract}

Results: There is no statistical significant correlation between measures 1, 2, 3 and 4 (n $=30)(\mathrm{p}>.05)$. To determine the level of correlation between the measures 1 and 3 and between measures 1 and 4 the Pearson Coefficient was used. However, the correlations were negative and close to zero (Measures 1 and 3:-0,069) (Measures 1 and 4: -0,103) and $P$ values > .05: (Measures 1 and 3: p 0,718) (Measures 1 and 4: $p$ 0,59). This information associated to $\mathrm{P}$ values greater than .05 indicates that no association between the measures seems to exist.

Conclusion: This current study, corroborating other ones 3,6 showed no correlations between nasal external measures and the amount of septal cartilage available for harvesting. Other methodologies should be developed and explored to guide the surgeon for a more precise surgical planning.

Keywords: grafting in rhinoplasty, nasal bone, harvesting, facial cosmetic surgery
Volume 6 Issue 4 - 2017

\author{
Renato Castro Alves de Sousa,' Gustavo \\ Figueiredo Nunes Rabelo, ${ }^{2}$ Henrique \\ Queiroz Correa Garchet, ${ }^{2}$ Mirian Cabral \\ Moreira de Castro ${ }^{2}$ \\ 'Chief of Facial Plastic Surgery Santa Casa de Belo Horizonte, \\ Brazil \\ ${ }^{2}$ Otorhinolaryngology Department Santa Casa de Belo \\ Horizonte, Brazil
}

Correspondence: Renato Castro Alves de Sousa Rua Padre Rolim 769 I2 Andar Belo Horizonte Minas Gerais, Brazil, Email renato@alvesdesousa.com.br

Received: November 17, 2016 | Published: April 06, 2017

\section{Introduction}

Rhinoplasty is a facial cosmetic surgery in which the structure of the nose is changed to obtain a better harmony with other facial components. It has been considered even by the greater specialists the most defiant procedure in facial plastic surgery. There is a vast amount of books and articles published in the medical literature related exclusive to this procedure, if you search for Rhinoplasty on Pubmed, more than 8000 articles will pull up. However, as basic principles remain unchanged, such as those relating the combination of an ideal nasal shape with an adequate function, new theories and techniques on this procedure are in constant development in order to achieve better and long-lasting results.

At the beginning, when the surgery was first developed, the surgical techniques were aggressive and based on more tissue resection to refine nasal regions. Therefore, the skin envelope was laid on a less structured nasal framework. ${ }^{1}$ Nowadays, the surgical techniques have changed prioritizing the tissue preservation. Surgeons have performed less tissue resection and used cartilaginous grafts to strengthen weak areas and reshape the underlying structures. This new concept of surgery is associated with long-lasting results and lesser functional and esthetic complication rates.
The autologous grafts have been frequently used to achieve the complexity demanded on this surgery. Among those grafts, septal, ear and costal cartilage are the ones most used. The septal cartilage is the graft of choice because is in the same surgical field, easily harvested, molded, and has more adequate characteristics considering shape, consistency and thickness, especially in primary cases. ${ }^{2}$ Therefore, a method capable of predicting the amount of septal cartilage available for grafting would be very useful to surgical planning and preoperative assessment.

The study of nasal anatomy is a frequent topic in the literature. ${ }^{1,2}$ However the majority of published papers deal with septoplasty techniques description and are deprived of more detailed anatomical studies. As to the best of our knowledge there is a scarcity of studies in the literature correlating the external nasal anatomy with the amount of septal cartilage available for grafting in Rhinoplasty. ${ }^{3}$ The main objective of our study is to evaluate if there is any correlation between the size of the nasal bone measured preoperatively and the amount of septal cartilage available for harvesting in the surgical field.

Kim et al., ${ }^{4}$ studied the nasal external measures and its correlation with the length of septal graft available for harvesting in humans. The study consisted in measuring the nasal external measures in 
open rhinoplasties and correlating them with the size and amount of septal cartilage available for harvesting. The external anthropometric measure showed no significant correlation with the septal area available for harvesting, indicating that even patients with larger external nasal dimensions might have little amount of septal cartilage available for harvesting. On the contrary, patients with short external nasal dimensions might have plenty of septal cartilage amount available for harvesting. ${ }^{5,6}$ As to the best of our knowledge, however, no paper was found in the literature correlating the specific size of nasal bone with the amount of septal cartilage available for harvesting. That correlation was the objective of our Study.

\section{Methods}

We selected 30 participants who presented for primary septorhinoplasty in our Outpatient Clinic (Hospital Santa Casa de Misericódia, Belo Horizonte, Brazil), and whose surgical planning included separation of the superior lateral cartilage from the septum to allow intraoperative measurement of the septal cartilage size. Data was collected from April 2014 to May 2015. The same surgeon (main author) performed all measures and procedures. Participants who underwent previous septorhinoplasty suffered some sort of nasal trauma resulting in nasal fracture or those whose surgical planning did not include the separation of the lateral cartilages from the septum were excluded from the study. All collected data were submitted to statistical analyses. This study was approved by the ethics committee of the Santa Casa de Belo Horizonte Hospital Research and Teaching Institute, Brazil. Participants signed a written informed consent.

\section{Surgical techinique}

All cases were performed via both endonasal and open approach to the nose. In the operating table, the main surgeon palpates the radix and rhinion and measures with a metric ruler the distance in millimeters between them (Figure 1). Afterwards, the surgeon palpates the anterior septal angle and measures its distance until the radix (Figure 2). The next step is to access the dorsum and dissect off the lateral cartilages from the septum. Then, the surgeon estimates the amount of septal cartilage available for harvesting through 2 measures. The distance in straight line from the rhinion to the anterior septal angle (Figure 3).

The distance in straight line from the osteo-cartilaginous transition to the caudal septal midpoint (Figure 4). Both measures were obtained inserting a Cottle instrumentith blue methylene on the limits of the proposed measures, before measuring it with a ruler.

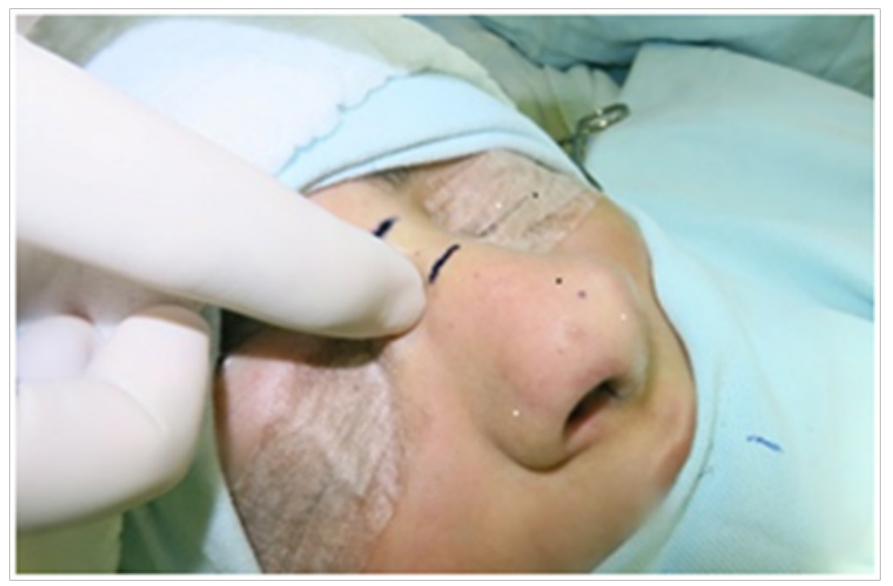

Figure I Measure I.

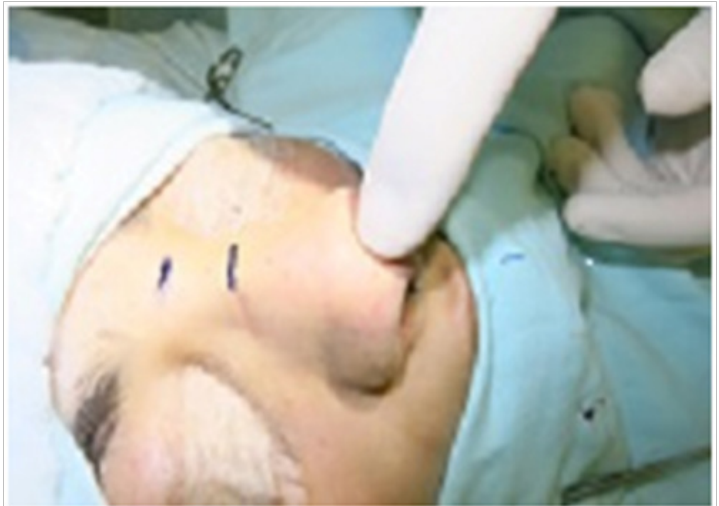

Figure 2 Measure 2.

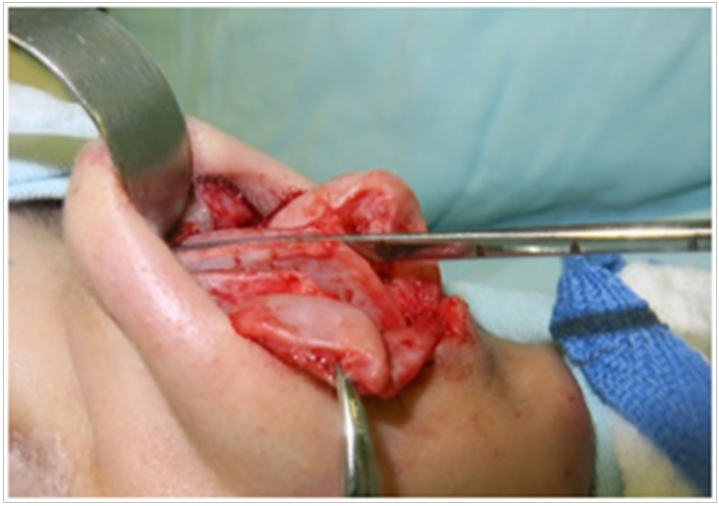

Figure 3 Measure 3.

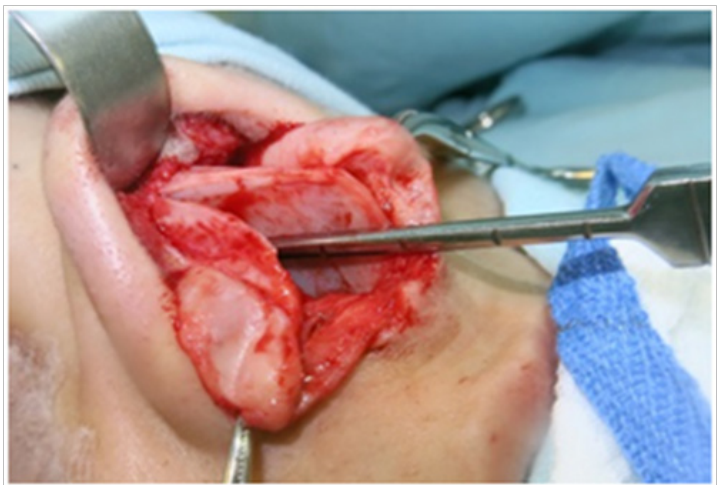

Figure 4 Measure 4.

\section{Statistical analysis}

In the statistical analysis of variables we calculated the correlation coefficient of Pearson (parametric) and Spearman (non-parametric) using the R Software 2.15 and Minitab 15. The assumption of normality was accessed by the Shapiro-Wilk test. Level of significance was $P$ $<.05$. Beyond the assessment of the correlation between measures $1,2,3$ and 4 , it was also evaluated the level of accuracy between the measure 2 and the sum of measures 1 and 3 , which are supposed to be coincident measures, using the $t$ test. The Intraclass Correlation Coefficient was also obtained to evaluate the level of agreement between measure 2 and the sum of measures 1 and 3 .

\section{Results}

The study included 30 patients 21 women (90\%) and 9 men 
$(30 \%)$. Patients ranged in age from 16 to 45 years, with a mean (SD) age of 27 years. The nasal bone mean size (measure 1) was $18.7 \mathrm{~mm}$ with a standard deviation of 4 and a median value of $18.5 \mathrm{~mm}$. The mean distance between the nasion and the anterior septal angle was $46.6 \mathrm{~mm}$ (measure 2), with a standard deviation of 4.9 and a median of $46.5 \mathrm{~mm}$. The mean distance in straight line from the caudal septum most superior point (anterior septal angle) to the rhinion was $26.4 \mathrm{~mm}$ (measure 3), with a standard deviation of $3.8 \mathrm{~mm}$ and a mean value of $25 \mathrm{~mm}$. The mean distance in straight line from the caudal septum midpoint to the osteo-cartilagenous transition area was $37.2 \mathrm{~mm}$, with a standard deviation of 4.2 and a median value of $38 \mathrm{~mm}$ (measure 4). Total nasal length (sum of measures 1 and 3 ) was a mean of 45.1 with a standard deviation of $5.4 \mathrm{~mm}$ and a median value of $44 \mathrm{~mm}$ (Table 1).

The mean proportion of the nasal bone (measure 1) relative to total nasal length (measures $1+3$ ), proportion 1 , was $41.4 \%$, with a Standard Deviation of 6.3 and a median of $41.8 \%$. The mean proportion of the measure in straight line from the most superior point of the caudal septum to the osteo-cartilagenous transition (measure 3 ) related to total nasal length (measures $1+3$ ), proportion 2 , was $58.6 \%$, with a Standard Deviation of $6.3 \%$ and a median of $58,2 \%$. The mean proportion of the measure in straight line from the caudal septum midpoint to the osteo-cartilagenous transition (measure 4) related to total nasal length (measures $1+3$ ), proportion 3 , and was $83.6 \%$, with a Standard Deviation of $14.3 \%$ and a median of $82.9 \%$ (Table 2).

To determine the level of correlation between the measures 1 and 3 and between measures 1 and 4 the Pearson Coefficient was used. However, the correlations were negative and close to zero. This information associated to $\mathrm{P}$ values greater than .05 indicates that no association between the measures seems to exist, in other words, the behavior of one measure does not influence the behavior of another one, as noticed in Table 3.

Table I Correlation between Measures

\begin{tabular}{|c|c|c|c|c|c|c|c|c|}
\hline Characteristcs & $\mathbf{N}$ & Mean & SD & Minimum & $I^{\circ} \mathbf{Q}$ & Median & $3^{\circ} \mathrm{Q}$ & Maximum \\
\hline Measure I (mm) & 30 & 18,7 & 4,0 & 12,0 & 15,0 & 18,5 & 21,3 & 27,0 \\
\hline Measure $2(\mathrm{~mm})$ & 30 & 46,6 & 4,9 & 34,0 & 44,8 & 46,5 & 50,0 & 55,0 \\
\hline Measure $3(\mathrm{~mm})$ & 30 & 26,4 & 3,8 & 20,0 & 23,0 & 25,0 & 30,0 & 35,0 \\
\hline Measure $4(\mathrm{~mm})$ & 30 & 37,2 & 4,2 & 30,0 & 33,0 & 38,0 & 40,0 & 46,0 \\
\hline Total nasal length $(\mathrm{I}+3)(\mathrm{mm})$ & 30 & 45,1 & 5,4 & 36,0 & 41,8 & 44,0 & 50,0 & 57,0 \\
\hline
\end{tabular}

N: Number of Patients; SD: Standard Deviation; $I^{\circ}$ Q: $1^{\circ}$ Quartile; $3^{\circ}$ Q: $3^{\circ}$ Quartile

Table 2 Correlation between Proportions

\begin{tabular}{lllllllll}
\hline & $\mathbf{N}$ & Mean & SD & Minimum & $\mathbf{I}^{\circ} \mathbf{Q Q}$ & Median & $\mathbf{3}^{\circ} \mathbf{Q}$ & $\mathbf{M a x i m u m}$ \\
\hline Proportion I (\%) & 30 & $4 I, 4$ & 6,3 & 27,9 & 37,7 & 41,8 & 47,2 & 50,0 \\
Proporção 2 (\%) & 30 & 58,6 & 6,3 & 50,0 & 52,8 & 58,2 & 62,3 & 72,1 \\
Proporção 3 (\%) & 30 & 83,6 & 14,3 & 58,2 & 77,6 & 82,9 & 93,0 & 124,3 \\
\hline
\end{tabular}

N: Number of Patients; SD: Standard Deviation; I ${ }^{\circ} \mathrm{Q}: I^{\circ}$ Quartile; $3^{\circ} \mathrm{Q}: 3^{\circ}$ Quartile

Table 3 Correlation between Measures

\begin{tabular}{lll}
\hline & Correlation Coefficient & P Value \\
\hline Measure I and Measure 3 & $-0,0691$ & 0,718 \\
Measure I and Measure 4 & $-0,1031$ & 0,590 \\
Measure 3 and Measure 4 & $-0,064 I$ & 0,739 \\
\hline
\end{tabular}

I: Pearson's Coefficient

The $\mathrm{T}$ test compares the mean of the differences to zero; it was used to compare the correlation between measure 2 and the sum of measures 1 and 3 . The calculated $p$ value $.032(<0.05)$ indicated that the difference between measure 2 and the sum of measures 1 and 3 is different from zero, indicating a statistically significant difference. The measure 2 is on average $1.5 \mathrm{~mm}$ greater than the sum of measures 1 and 3 (95\% confidence interval of 13 to 2.8$)$, which may be attributed to the difference in precision on how each measure was obtained, measure 2 was obtained through palpation of its limits, measure 3 was obtained through direct measuring with a metric ruler.

\section{Conclusion}

Our theory was that patients with shorter nasal bones would have greater amounts of septal cartilage available for harvesting. However, no statistical significant correlation was found in our study between the size of the nasal bone (measure 1) and amount of septal cartilage available for harvesting estimated by 2 measures: the distance in straight line from the caudal septum most superior point (anterior septal angle) to the rhinion (measure 3 ) and the mean distance in straight line from the caudal septum midpoint to the osteo cartilagenous transition (measure 4). Statistical analysis showed that the behavior of measure 1 does not affect the behavior of measures 3 and 4 . Those findings are in accordance with previous studies. ${ }^{3-6}$

Predicting the amount of cartilage available for harvesting is still a challenge for most Rhinoplasty Surgeons. None of the current studies was able to make any correlations between nasal external measures and the amount of septal cartilage available for harvesting. Therefore, other methodologies should be developed and explored to guide the surgeon for a more precise surgical planning in order to allow the use of available grafts in a more effective way.

\section{Acknowledgments}

None.

\section{Conflicts of interest}

Author declares there bare no conflicts of interest.

\section{Funding}

None.

\section{References}

1. Dingman RO, Natvig P. Surgical anatomy and aesthetics in corrective rhinoplasty. Clin Plast Surg. 1977;4(1):111-112.

2. Godley FA. Nasal septal anatomy and its importance in septal reconstruction. Ear Nose Throat J. 1997; 76(8): 498-506 
3. Wang H, Fan F, Wang S, et al. Intraoperative measurements of harvestable septal cartilage in rhinoplasty. Ann Plast Surg. 2011; 67(2):201.

4. Kim JS, Han KH, Choi TH, et al. Correction of the nasal tip and columella in Koreans by a complete septal extension graft using an extensive harvesting technique. J Plast Reconstr Aesthet Surg. 2007;60(2):163-170.

5. Mowlavi A, Masouem S, Kalkanis J, et al. Septal cartilage defined: implications for nasal dynamics and rhinoplasty. Plast Reconstr Surg. 2006;117(7):2171-2174.

6. Miles BA, Petrisor D, Kao H, et al. Anatomical variation of the nasal septum: analysis of 57 cadaver specimens. Otolaryngol Head Neck Surg. 2007;136(3):362-368
7. Sheen J. Aesthetic Rhinoplasty. The CV Mosby Co, St. Louis, USA. 1978.

8. Camirand A, Doucet J, Harris J. Nose surgery: how to prevent a middle vault collapse - a review of 50 patients 3 to 21 years after surgery. Plast Reconstr Surg. 2004;114(2):527-534.

9. Johnson CM, Toriumi DM. Open structured rhinoplasty. Philadelphia: WB Saunders Co, USA. 1990. 